PHYSICAL REVIEW D 89, 091501(R) (2014)

\title{
Impact-parameter analysis of TOTEM data at the LHC: Black disk limit exceeded
}

\author{
A. Alkin and E. Martynov \\ Bogolyubov Institute for Theoretical Physics, Metrologichna 14b, Kiev UA-03680, Ukraine \\ O. Kovalenko \\ Taras Shevchenko Kiev National University, Volodimirska 60, Kiev UA-03101, Ukraine
}

S. M. Troshin

Institute for High Energy Physics, Protvino, Moscow Region 142281, Russia

(Received 1 April 2014; revised manuscript received 21 April 2014; published 16 May 2014)

\begin{abstract}
We discuss the profile of the impact-parameter dependent elastic scattering amplitude. Extraction of impact-parameter dependence from the data set with inclusion of the experimental data on elastic scattering at the LHC energies helps to reveal the asymptotics of hadron interactions. Analysis of the data clearly indicates that the impact-parameter elastic scattering amplitude exceed the black disk limit at the LHC energy $7 \mathrm{TeV}$ and the inelastic overlap function reaches its maximum value at $b>0$.
\end{abstract}

DOI: 10.1103/PhysRevD.89.091501

\section{INTRODUCTION}

We show here that using the data on an elastic differential cross section can provide new information for the asymptotics of hadron scattering. Of particular importance is the extraction of the impact-parameter dependent quantities from this experimental data including the recent measurement at LHC energies.

One of the attractive features of the impact parameter representation is a diagonalization of the unitarity equation for the elastic scattering amplitude $H(s, b)$, i.e. at high energies,

$$
\operatorname{Im} H(s, b)=|H(s, b)|^{2}+G_{\text {inel }}(s, b),
$$

with $\mathcal{O}(1 / s)$ precision [1]. The term $|H(s, b)|^{2}$ is the elastic channel contribution, $G_{\text {inel }}(s, b)$ covers all the intermediate inelastic channels, and $b$ is an impact parameter of the colliding hadrons.

Information on $H(s, b)$, in particular, on $H(s, 0)$, is necessary to select the upper limit for this amplitude, namely, to know if this limit should be one half (it is the black disk limit) and correspond to the maximum of the inelastic channel contribution to the elastic unitarity with asymptotic ratio

$$
\sigma_{\mathrm{el}}(s) / \sigma_{\mathrm{tot}}(s) \rightarrow 1 / 2
$$

or if it is equal to unity and corresponds to a maximal value of the partial amplitudes allowed by unitarity resulting in the limit

$$
\sigma_{\mathrm{el}}(s) / \sigma_{\mathrm{tot}}(s) \rightarrow 1
$$

at $s \rightarrow \infty$. Under assumption of the limit $1 / 2$ for the partial amplitude, the factor in the original Froissart-Martin bound
PACS numbers: 13.85.-t, 13.85.Dz, 13.85.Lg, 29.85.Fj

for the total cross sections has been reduced by 2 [2]. The bound reduced by factor of 4 for the total inelastic cross section has also been derived [3]. Several asymptotic limits have been treated in [4] in an almost model-independent way, but also for the forward scattering data only.

As well, Eq. (1) is instrumental for the reconstruction of $G_{\text {inel }}(s, b)^{1}$ from the elastic scattering data. ${ }^{2}$

The unitarity relation implies existence of the two scattering modes, designated as absorptive and reflective. Namely, the elastic scattering $\tilde{S}$-matrix element [related to the elastic scattering amplitude as $\tilde{S}(s, b)=1+2 i H(s, b)]$ can be presented in the form

$$
\tilde{S}(s, b)=\kappa(s, b) \exp [2 i \delta(s, b)]
$$

with the two real functions $\kappa(s, b)$ and $\delta(s, b)$. The function $\kappa(0 \leq \kappa \leq 1)$ is an absorption factor, ${ }^{3}$ its value $\kappa=0$ corresponds to a complete absorption. At high enough energies the real part of the scattering amplitude can be neglected, allowing the substitution $H \rightarrow i H$. We consider this simplified case for the moment here. The choice of elastic scattering mode, namely, absorptive or reflective, is governed by the phase $\delta(s, b)$. The common assumption is that $\tilde{S}(s, b) \rightarrow 0$ at the fixed impact parameter $b$ and $s \rightarrow \infty$. It is called a black disk limit and the elastic scattering in this case is completely absorptive, i.e. it is just a shadow of all the inelastic processes. This implies $\max \{\operatorname{Im} H(s, b)\}=1 / 2$.

\footnotetext{
${ }^{1}$ The inelastic overlap function $G_{\text {inel }}(s, b)$ is not well suited for asymptotics studies.

${ }^{2}$ Compare, e.g., [5,6] for an earlier analysis of $G_{\text {inel }}(s, b)$ and [7] for the most recent one.

${ }^{3}$ It has different meaning in the reflection region, as it will be discussed further.
} 
There is another possibility, namely, the function $\tilde{S}(s, b) \rightarrow-1$ when $b$ is fixed and $s \rightarrow \infty$, i.e. $\kappa \rightarrow 1$ and $\delta \rightarrow \pi / 2$. This case corresponds to a pure reflective scattering [8]. The principal point is that the phase is nonzero, i.e. $\delta$ is equal to $\pi / 2$ and $\max (\operatorname{Im} H(s, b))=1$.

We discuss now the observable effects sensitive to the presence of the nonzero phase. The most straightforward way is to extract impact-parameter dependent elastic scattering amplitude from the experimental data for the $p p$ and $\bar{p} p$ scattering.

\section{IMPACT ANALYSIS OF THE DATA}

Impact parameter analysis is performed following (with a minor modification) the method suggested by Amaldi and Schubert [5] for $p p$ scattering and applied by Fearnley [6] to $\bar{p} p$ scattering. Let us shortly describe how the amplitudes in impact parameter representation were extracted in ama, fearn from the measured $d \sigma / d t$. We start with the relation between the impact $H(s, b)$ and standard $A(s, t)$ amplitudes ( $b$ is given in $f m$ ):

$$
\begin{aligned}
H(s, b) & =\frac{1}{8 \pi s} \int_{0}^{\infty} d q q J_{0}\left(q b / k_{1}\right) A(s, t), \\
A(s, t) & =8 \pi s \int_{0}^{\infty} d b b J_{0}\left(q b / k_{1}\right) H(b, s), \\
t & =-q^{2},
\end{aligned}
$$

$k_{1}=0.1973269718 \mathrm{GeV}$ fm. Normalization of $A(s, t)$ is the following (total cross section is measured in $m b$ ):

$$
\sigma_{t}=\frac{k_{2}}{s} \operatorname{Im} A(s, 0), \quad \frac{d \sigma}{d t}=\frac{k_{2}}{16 \pi s^{2}}|A(s, t)|^{2},
$$

where $k_{2}=0.389379338 \mathrm{mb} \mathrm{GeV}^{2}$.

To describe the data on $d \sigma / d t$ we used parametrizations of $A(t) \equiv A(s, t))$ (at fixed energy) modified from those in $[5,6]$ :

$$
\begin{aligned}
A(t)= & 8 \pi s\left\{i \alpha(1-i \rho)\left(A_{1} e^{b_{1 \alpha} t / 2}+\left(1-A_{1}\right) e^{b_{2 \alpha} t / 2}\right)\right. \\
& \left.-i A_{2} e^{b_{3} t / 2}-A_{2} \rho(1-t / \tau)^{-4}\right\},
\end{aligned}
$$

where

$$
\alpha=(1-i \rho)\left(\sigma_{t} /\left(8 k_{2} \pi\right)+A_{2}\right),
$$

$\rho$ and $\sigma_{t}$ are the experimental values of real to imaginary part ratio of amplitude at $t=0$ and total cross section (in $\mathrm{mb})$ at the given energy. Parameters were fitted at each energy. We would like to emphasize that the parametrization (6) is constructed in such a way that it obeys $\operatorname{Im} A(s, 0)=8 \pi g s \sigma_{t}$ and $\operatorname{Re} A(s, 0) / \operatorname{Im} A(s, 0)=\rho$ with any values of the parameters.

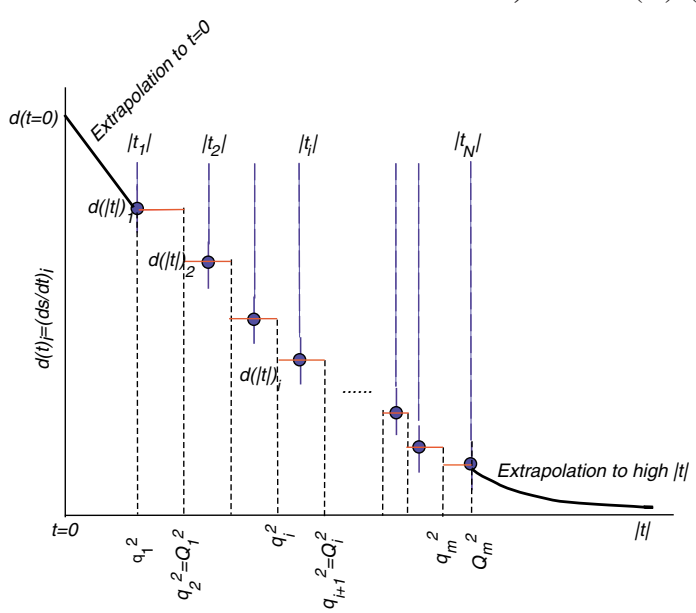

FIG. 1 (color online). Scheme of bins in Eqs. (8) and (9).

\section{A. Imaginary part of impact elastic scattering amplitude}

$H(s, b)^{4}$ is calculated at each considered (and fixed) energy as

$$
\operatorname{Im} H^{d}(b)=\frac{1}{8 \pi s} \sum_{i=1}^{N} \int_{q_{i}}^{Q_{i}} d q q J_{0}\left(\frac{b q}{k_{1}}\right) I(q)_{i},
$$

where $N$ is number of points in the $d \sigma / d t$ data set at given energy,

$$
I(q)_{i}=\sqrt{\left(16 \pi s^{2} / k_{2}\right)(d \sigma / d t)_{i}-(\operatorname{Re} A)_{i}^{2}},
$$

and $(d \sigma / d t)_{i}$ is the experimental value of $d \sigma / d t$ measured at $t=t_{i}$ while $(\operatorname{Re} A)_{i}$ is the real part of the amplitude parametrized in the form (6) and calculated at $t=t_{i}$. The boundaries $q_{i}, Q_{i}$ of the $i$ th bin are defined as

$$
\begin{aligned}
q_{i}^{2} & =\left|t_{i}-t_{i-1}\right| / 2, \\
Q_{i}^{2} & =\left|t_{i+1}-t_{i}\right| / 2 .
\end{aligned}
$$

Extrapolations to low and high $t$ were considered separately. Figure 1 describes the entire scheme.

In the region $0 \leq|t| \leq\left|t_{1}\right|$ the following extrapolation has been used:

$$
A^{(\text {low })}(t)=i A_{0} \exp \left(-B_{0}|t|\right)+\operatorname{Re} A(t) / 8 \pi s,
$$

where the real part of amplitude $\operatorname{Re} A(t)$ is to be taken from the initial parametrization (6). The constant $A_{0}$ can be found from the optical theorem

$$
A_{0}=s k_{2} / \sigma_{t}
$$

\footnotetext{
${ }^{4}$ The profile function $\Gamma(s, b)=-2 i H(s, b)$ has been extracted from the data in $[5,6])$.
} 
Here $\sigma_{t}$ is the experimental value of the total cross section at given energy. The slope $B_{0}$ is determined from the continuity condition at the first experimental point $t=t_{1}$,

$$
\frac{d \sigma\left(t=t_{1}\right)}{d t}=\frac{k_{2}}{16 \pi s^{2}}\left|A^{(\text {low })}\left(t_{1}\right)\right|^{2} .
$$

So, for lower $|t|$ values one can write $\left(t=-q^{2}\right)$

$$
\operatorname{Im} H^{(\text {low })}(b)=\frac{1}{16 \pi s^{2}} \int_{0}^{\left|t_{1}\right|} d q q J_{0}\left(\frac{b q}{k_{1}}\right) \operatorname{Im} A^{(\text {low })}(t) .
$$

Thus

$$
\operatorname{Im} H(b)=\operatorname{Im} H^{(\text {low })}(b)+\operatorname{Im} H^{(d)}(b)+\operatorname{Im} H^{(\text {high })}(b) .
$$

It can be shown that extrapolation to higher $|t|, \operatorname{Im} H^{(\text {high) }}(b)$ is negligible with any form of parametrization.

\section{B. Uncertainty calculation}

As the quantity under consideration depends on the data in a rather complicated way, uncertainties from the experimental points were propagated numerically by varying those within their respective limits (assuming the quoted uncertainty to be $\sigma$ interval) which produced a set of results for $\operatorname{Im} H(b)$. The standard deviation of the resulting values of $\operatorname{Im} H(b)$ at a given $b$ point was used as an uncertainty estimate.

The real part of $H(b)$ is computed according to the equation

$$
\operatorname{Re} H(b)=\frac{1}{8 \pi s} \int_{0}^{\infty} d q q J_{0}\left(\frac{b q}{k_{f m}}\right) \operatorname{Re} A(q) .
$$

The standard error propagation formula can be used in this case. An error can be defined as

$$
\delta \operatorname{Re} H(b)=\sqrt{\frac{\partial \operatorname{Re} H(b)}{\partial p_{i}} \frac{\partial \operatorname{Re} H(b)}{\partial p_{j}} V_{i j}} .
$$

Covariance matrix $V_{i j}$ for parameters $p_{i}$ of the parametrization (6) was taken from minimization procedure (MINUIT).

\section{The results}

We have analyzed data on $p p$ elastic scattering at $\sqrt{s}=$ $23.5,30.7,44.7,52.8,62.5,7000 \mathrm{GeV}[5,9,10]$ and $\bar{p} p$ at $\sqrt{s}=53,546,1800-1960 \mathrm{GeV}$ [11-15]. The data at $|t| \geq$ $0.1 \mathrm{GeV}^{2}$ were used for analysis. The main goal of our analysis is to extract $\operatorname{Im} H(b)$ from the TOTEM data at $\sqrt{s}=7 \mathrm{TeV}$ [10]. However in order to check the method we have applied it to older data to cross-check with $[5,6]$. We have found that our results for energies of the old accelerators ISR, SPS (at CERN) and Tevatron (at Fermilab) are compatible with those in $[5,6]$. A detailed

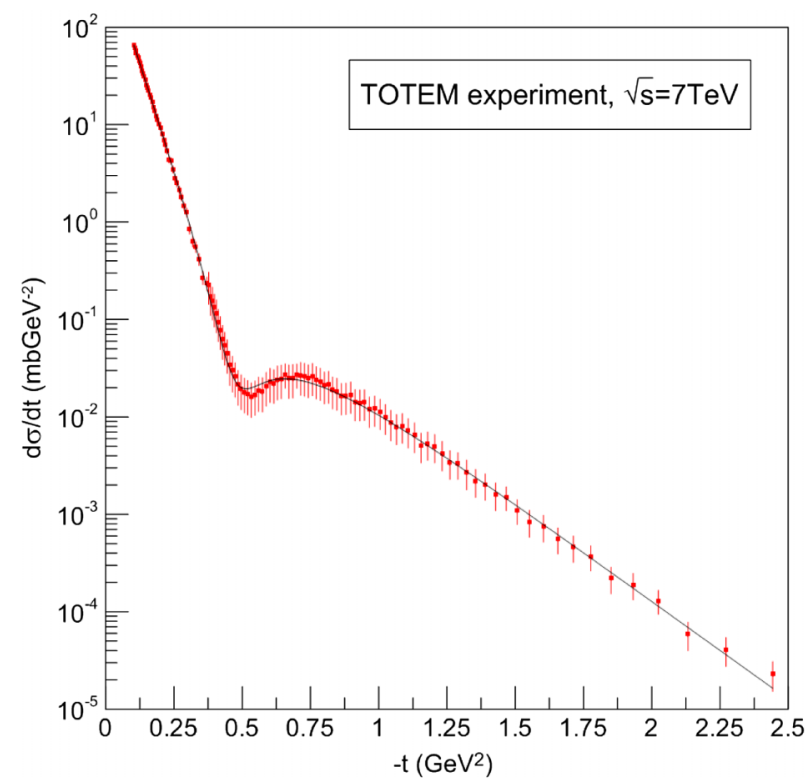

FIG. 2 (color online). Description of the TOTEM data at $7 \mathrm{TeV}$ with the amplitude parametrization (6).

explanation of our analysis will be presented in a separate paper. Here we demonstrate the main results of our analysis, shown in Figs. 2-4(b). Figure 2 illustrates a quality $\left(\chi^{2} / d f \approx 0.15\right)$ of the TOTEM data description [the values of parameters in Eq. (6) are given in Table I] while the results of our impact analysis for $\operatorname{Im} H(b), \operatorname{Re} H(b)$ and $G_{\text {inel }}(b)$ at $\sqrt{s}=7 \mathrm{TeV}$ are presented in Fig. 3. To verify a self-consistency of the method we have calculated the value of $\rho$ from the extracted impact amplitudes

$$
\rho(s)=\frac{\int_{0}^{b_{\max }} d b b \operatorname{Re} H(s, b)}{\int_{0}^{b_{\max }} d b b \operatorname{Im} H(s, b)}
$$

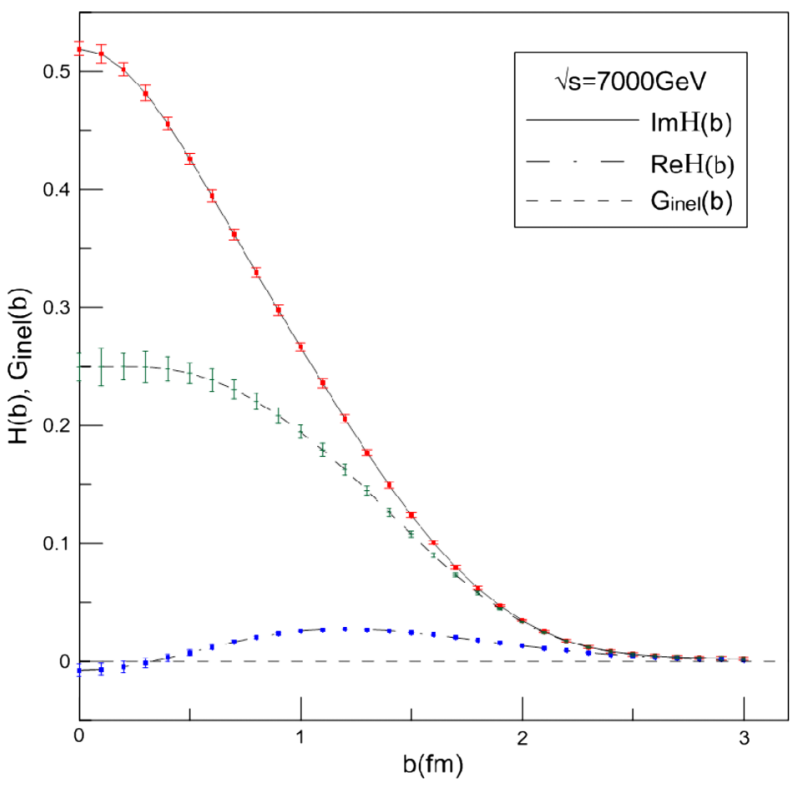

FIG. 3 (color online). Impact $p p$ amplitudes at $7 \mathrm{TeV}$. 


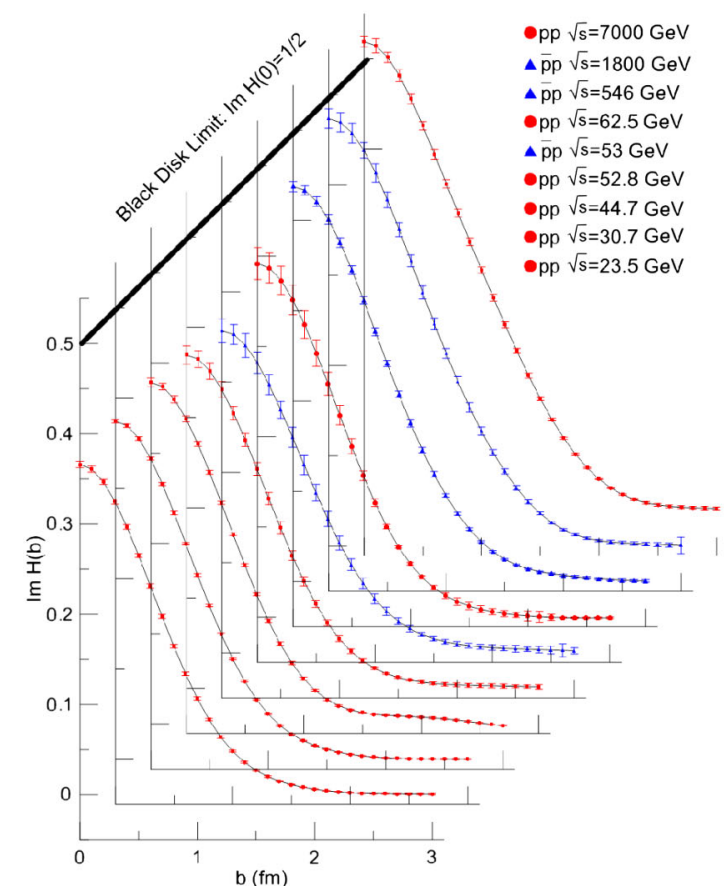

(a) $\operatorname{Im} H(s, b)$

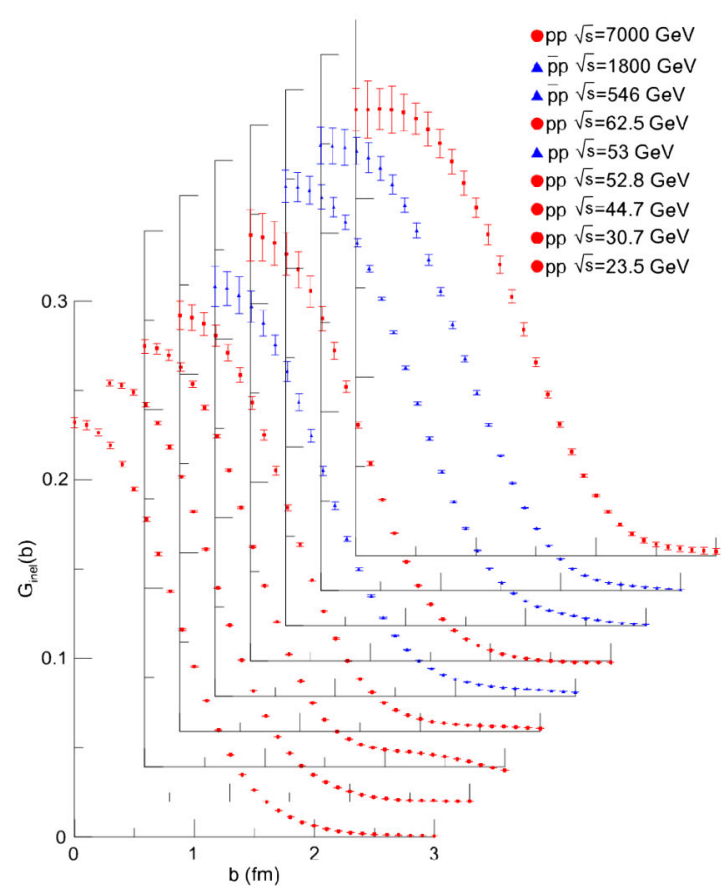

(b) $\mathrm{G}_{\text {inel }}(s, b)$

FIG. 4 (color online). Imaginary parts of impact amplitudes and inelastic overlap functions of $p p$ and $\bar{p} p$ at various energies.

at $\sqrt{s}=7 \mathrm{TeV}$ and with $b_{\max }=3 \mathrm{fm}$ we obtained $\rho \approx 0.138$, which corresponds to the "experimental" value $\rho=0.14$ used by TOTEM in [10]. There is no sense to extend the integration limits to higher $b_{\max }$ because the available data on $d \sigma / d t$ produce too big errors in $\operatorname{Im} H(s, b)$ and $\operatorname{Re} H(s, b)$. However it is quite evident that a contribution of high $b$ 's should be small and will improve the result.

The most impressive fact is that $\operatorname{Im} H(b)>1 / 2$ at small $b$. As was expected the $\operatorname{Re} H(b)$ is quite small. In Figs. 4(a) and 4(b) the evolution of $\operatorname{Im} H(b)$ and $G_{\text {inel }}(b)$ is presented.

\section{DISCUSSION OF RESULTS}

Now we proceed to the qualitative implications of the discussed results. A gradual transition to the reflective scattering mode occurs in the models where elastic

TABLE I. Parameters of the model Eq. (6) obtained by fitting to the TOTEM data at $\sqrt{s}=7 \mathrm{TeV}$.

\begin{tabular}{lcc}
\hline \hline Parameters & Values & Errors \\
\hline$A_{1}(\sqrt{m b} / \mathrm{GeV})$ & 0.120 & \pm 0.069 \\
$A_{2}(\sqrt{m b} / \mathrm{GeV})$ & 1.166 & \pm 0.301 \\
$b_{1}\left(\mathrm{GeV}^{-2}\right)$ & 0.442 & \pm 0.121 \\
$b_{2}\left(\mathrm{GeV}^{-2}\right)$ & 0.863 & \pm 0.037 \\
$b_{3}\left(\mathrm{GeV}^{-2}\right)$ & 4.637 & \pm 0.284 \\
$\tau\left(\mathrm{GeV}^{2}\right)$ & 0.589 & \pm 0.277 \\
$\sigma_{t}(\mathrm{mb})$ & 98.3 & Fixed \\
$\rho$ & 0.14 & Fixed \\
\hline \hline
\end{tabular}

scattering $\tilde{S}$-matrix element $\tilde{S}(s, b)$ passes through zero to the negative values with increasing energy. This transition implies appearance of the phase $\delta=\pi / 2$. The solution of the equation $\tilde{S}(s, b)=0$ separates the regions of absorptive and reflective scattering and corresponds to the maximum value of $G_{\text {inel }}(s, b)=1 / 4$ since the derivative of $G_{\text {inel }}(s, b)$ has the form

$$
\frac{\partial G_{\text {inel }}(s, b)}{\partial b}=\tilde{S}(s, b) \frac{\partial H(s, b)}{\partial b}
$$

and equals zero at $\tilde{S}(s, b)=0$. The derivative of the inelastic overlap function has the sign opposite to the sign of $\partial H(s, b) / \partial b$ in the region where $\tilde{S}(s, b)<0$ and the nonzero phase is, therefore, responsible for the transformation of the central impact-parameter profile of the function $H(s, b)$ into a peripheral one of the inelastic overlap function $G_{\text {inel }}(s, b)$. It can also be easily seen by expressing the function $G_{\text {inel }}(s, b)$ as a product, i.e.,

$$
G_{\text {inel }}(s, b)=H(s, b)(1-H(s, b)) .
$$

If $H(s, b)>1 / 2$ at high energy and small impact parameters, then the function $G_{\text {inel }}(s, b)$ will have a maximum value of $1 / 4$ at the nonzero impact parameter value. We found some weak indication of such a maximum at $\sqrt{s}=7 \mathrm{TeV}$. In Fig. 5 one can see that central values of the extracted $G_{\text {inel }}(s, b)$ data have a very shallow maximum at small $b$. The values of $G_{\text {inel }}(s, b)$ at some $b$ point are also given in the figure. It would be interesting to see if this peak will be more pronounced at higher energies. 
IMPACT-PARAMETER ANALYSIS OF TOTEM DATA AT ...

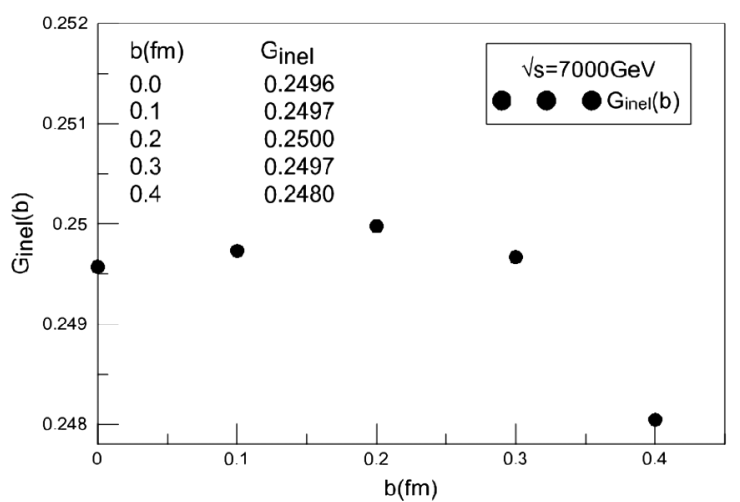

FIG. 5. The values of function $G_{\text {inel }}(b)$, extracted from the TOTEM data at small $b$.

It should be noted that the derivative of the elastic overlap function has no sign-changing factor in front of $\partial H(s, b) / \partial b$, namely,

$$
\frac{\partial G_{e l}(s, b)}{\partial b}=[1-\tilde{S}(s, b)] \frac{\partial H(s, b)}{\partial b}
$$

with $1-\tilde{S}(s, b)$ being non-negative at all values of $s$ and $b$.
PHYSICAL REVIEW D 89, 091501(R) (2014)

The role of the nonzero phase in the high energy scattering is essential. In the presence of the nonzero phase at the LHC energies the reflective scattering dominates at small impact parameters while inelastic processes are peripheral. The albedo (coefficient of reflection) increases with energy at $s>s_{0}$ [8]. The factor $\kappa(s, b)$ plays the role of albedo at $s>s_{0}$ and $b<R(s)$ and hence should be considered a reflective rather than absorption factor in this region.

Thus, the present analysis helps to understand which scattering mode is realized in asymptotics. Namely, assuming a monotonous energy dependence of the elastic scattering amplitude at the LHC energies and beyond one can conclude that reflective scattering mode is preferable on the basis of this analysis which demonstrates that the elastic scattering amplitude exceeds the black disk limit at $\sqrt{s}=7 \mathrm{TeV}$. The near-future measurements of elastic scattering at the LHC energies $\sqrt{s}=10-13 \mathrm{TeV}$ are very interesting and important for the confirmation or disproval of the above conclusion.

\section{ACKNOWLEDGMENTS}

We are grateful to N. E. Tyurin and G. M. Zinovjev for the many interesting discussions.
[1] M. L. Goldberger and K. M. Watson, Collision Theory (John Wiley and Sons, Inc., New York, 1964).

[2] A. Martin and S. M. Roy, Phys. Rev. D 89, 045015 (2014), and references therein.

[3] T. T. Wu, A. Martin, S. M. Roy, and V. Singh, Phys. Rev. D 84, 025012 (2011).

[4] D. A. Fagundes and M. J. Menon, Nucl. Phys. A880, 1 (2012).

[5] U. Amaldi and K. S. Schubert, Nucl. Phys. B166, 301 (1980).

[6] T. Fearnley, CERN, Report No. CERN-EP-85-137, 1985.

[7] I. M. Dremin and V. A. Nechitailo, Nucl. Phys. A916, 241 (2013).
[8] S. M. Troshin and N. E. Tyurin, Int. J. Mod. Phys. A 22, 4437 (2007).

[9] E. Nagy et al.Nucl. Phys. B150, 221 (1979).

[10] G. Antchev et al. (TOTEM Collaboration), Europhys. Lett. 101, 21002 (2013).

[11] A. Breakstone et al., Nucl. Phys. B248, 253 (1984).

[12] M. Bozzo et al., Phys. Lett. 155B, 197 (1985).

[13] N. A. Amos et al. (E-710 Collaboration), Phys. Lett. B 247, 127 (1990).

[14] F. Abe et al. (CDF Collaboration), Phys. Rev. D 50, 5518 (1994).

[15] V. M. Abazov et al. (D0 Collaboration), Phys. Rev. D 86, 012009 (2012). 